\title{
Fabrication of Si-PDMS Low Voltage Capillary Electrophoresis Chip
}

\author{
Wenwen GU ${ }^{1,2,3,4}$, Zhiyu WEN ${ }^{1,2,3,4}$, Zhongquan WEN ${ }^{1,2,3,4}$, Yi XU ${ }^{1,2,3}$, \\ Fengfei LIANG ${ }^{1,2,3}$, Xiaoguo HU $\mathrm{HU}^{1,2,3}$ \\ ${ }^{1}$ Key Laboratory of Fundamental Science of Micro/Nano-Device and System Technology, Chongqing, China \\ ${ }^{2}$ National Center for International Research of Micro/Nano-System and New Material Technology, Chongqing, China \\ ${ }^{3}$ Microsystem Research Center, Chongqing University, Chongqing, China \\ ${ }^{4}$ College of Optoelectronic Engineering, Chongqing University, Chongqing, China \\ Received May 11, 2009; revised July 2, 2009; accepted July 10, 2009
}

\begin{abstract}
This paper discusses the fabrication of Si-PDMS low voltage capillary electrophoresis chip (CE chip). Arrayed-electrode which is used to apply low separation voltage is fabricated along the sidewalls of the separation channel on the silicon based bottom part. Isolation trenches, which are placed surrounding the arrayed-electrode, insure the insulation between the arrayed-electrode, as well as arrayed-electrode and liquid in the micro channel. Polydimethylsilicone (PDMS) is used as the cover. PDMS and silicon based bottom part are reversible sealed to attain Si-PDMS low voltage CE chip. Experiments have been done to obtain optimum electrophoresis separation condition: separation voltage is $45 \mathrm{~V}$, switch time is $2 \mathrm{~s}$ and the Phe and Lys electrophoresis separation is successful.
\end{abstract}

Keywords: Low Voltage, Arrayed-Electrode, Si-PDMS, CE Chip

\section{Introduction}

Lab on a Chip, or called Miniaturized Total Analysis System ( $\mu$-TAS [1]), which has features of miniature, fast, high performance and throughput, has become a hot research topic in analytical chemistry. Capillary Electrophoresis Chip (CE chip) is a highly integrated miniature separation and analysis device within the development of biotechnology and MEMS (Micro-Electro-Mechanic Systems) technology. It can integrate sample treatment, injection, separation and detector into a microchip which is only several square centimeters. In CE chip, micro channels form network, and controlled microfluidic runs through all the system [2]. With the development of CE chip and widely applications, many fields such as disease diagnosis, environmentent monitoring, new drug development, food safety inspection will be changed thoroughly. Nowadays, it has already become one of the most important study subjects in chemistry, life science, MEMS, physics, micro-electronics and etc [3,4].

Taking a wide view of the related research status [5-8], working voltage which is from several hundreds to thousands is directly applied in the two ends of the separation channel of the CE chip. There are some shortages bought by high voltage, such as huge equipments, high thermal effect and high safety protection requirements for laboratory operators. High voltage also restricts the miniaturization, home application and portability for CE chip.

According to the moving gradient electrical field theory of CE chip, the separation channel can be equivalent to series connections of several shorter ones. Using arrayed-electrode to apply low separation voltage section by section can obtain the demanded high separation electric field. So the difficult problem brought by CE chip requiring high separation voltage has been overcome [9-12].

Based on the idea above, a new approach has been proposed to fabricate low voltage CE chip. Silicon-oninsulator (SOI) wafer is used as the substrate material of the silicon based bottom part, where there is a cross channel and arrayed-electrode. Arrayed-electrode and isolation trenches are fabricated along the micro channel 
sidewalls. The arrayed-electrode is used to apply low separation voltage. The isolation trenches insure the insulation between the arrayed-electrode, as well as arrayed-electrode and liquid in the micro channel. PDMS (Polydimethylsilicone) is used as the cover of the CE chip. The low voltage CE chip is obtained by reversible sealing the silicon based bottom part and the PDMS cover.

\section{Low Voltage Separation Principle}

The length of separation channel for conventional CE chip is several centimeters, and tens of kilovolts voltage must be applied in two ends of the separation channel. According to the principle of electrophoresis separation and the requirement of invariable electric field intensity in the separation channel, we proposed a thought of applying voltage section by section and circularly in the separation channel. Therefore, we design and fabricate arrayed-electrode with equal interval in the micro channel and periphery controlling circuit to control the time and range of the voltage on the arrayed-electrode. Figure 1(a) shows the model of low voltage separation. During the electrophoresis separation process, voltage is firstly applied on the first and third arrayed-electrode to drive sample move for a period time $T$ (we call it switch time). Then, voltage is applied on the second and forth arrayed-electrode for the same switch time. Next, voltage is applied on the rest of the arrayed-electrode which is followed this rule until the final pair of arrayed-electrode is affected. After the first cycle is finished, it goes to the next cycle. Voltage begins to apply on the first and third arrayed-electrode again. From Figure 1(a), it is obvious that the method for applying voltage is totally depended on the switch time and voltage applied on the arrayed-electrode. Therefore, we can select and optimize the separation condition to obtain the best result.

Figure 1(b) shows the control system for low voltage separation. The main electronic components are Microprogrammed Control Unit (MCU), communication interface, current amplifier circuit and array relay. Computer sends orders to MCU, which controls time sequence of voltage applied to every arrayed-electrode. $\mathrm{MCU}$ is used to control array-relay to carry out one of the three states of ground, high level or suspending on the arrayed-electrode. After the electrophoresis separation is finished, the experiment results will return to MCU, and be displayed in monitor screen.

\section{Fabrication Process}

\subsection{Layout Design}

Based on the electric and flow field simulating results of

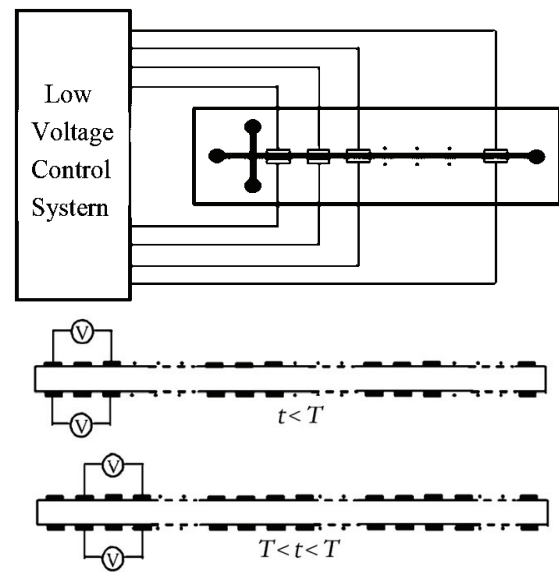

(a)

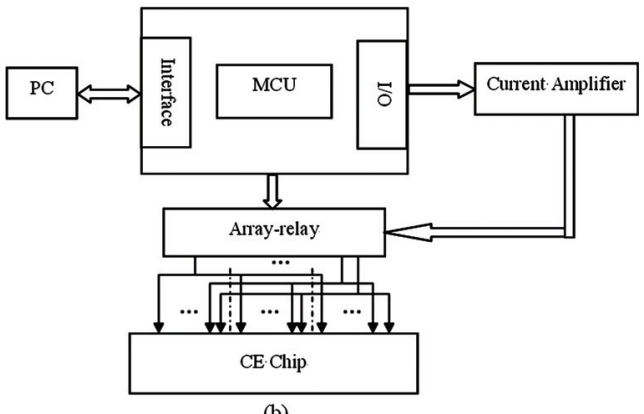

(b)

Figure 1 Schematic diagram of low voltage separation (a) model of low voltage separation; (b) control system.

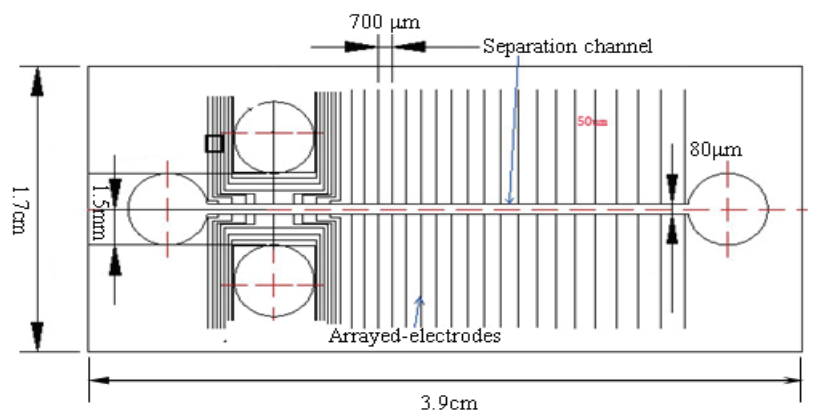

Figure 2. Structure diagram of the low voltage CE chip.

low voltage CE chip $[13,14]$, the structure of the CE chip has been designed, and the geometry parameters have been determined. The low voltage CE chip contains two parts: one is the silicon based bottom part where there is a cross microchannel and arrayed-electrode, and the other is PDMS cover where there are four reservoirs. The size of the designed CE chip is $3.9 \mathrm{~cm} \times 1.7 \mathrm{~cm}$, where the separation channel is $3 \mathrm{~cm}$ in length, $80 \mu \mathrm{m}$ in width, and $15 \mu \mathrm{m}$ in depth and the injection channel is $6 \mathrm{~mm}$ in length, $40 \mu \mathrm{m}$ in width, $15 \mu \mathrm{m}$ in depth. Along the sidewalls of the injection and separation channel, there are respectively 8 and 39 pairs of arrayed-electrode between which the interval is $700 \mu \mathrm{m}$. The specific dimensions of the low voltage CE chip are showed in Figure 2. 


\subsection{Silicon Based Bottom Part of Low Voltage CE Chip}

How to fabricate arrayed-electrode is the key point of successful electrophoresis for low voltage CE chip. Besides, how to insulate voltage and current between arrayed-electrode is also very important. This paper proposes using narrow channel deep trough etching [15] and polysilicon refilling, so arrayed-electrode can be insulated to isolated islands completely [16]. Then, arrayedelectrode is formed by ions diffusion. We select SOI (Silicon on Insulation) wafer as the substrate of the low voltage $\mathrm{CE}$ chip, because the influence to device layer caused by substrate (block effect) is reduced, as well as the parasitic effect caused by silicon device. Figure 3 shows processing scheme of silicon based bottom part of low voltage CE chip on SOI substrate.

1) Oxidize on the SOI wafer (device layer $15 \mu \mathrm{m}$ ) to form a $\mathrm{SiO}_{2}$ shielding layer with the thickness of $400-600 \mathrm{~nm}$ as the insulating layer of the bottom of micro arrayed-electrode; 2) Photoetch isolation regions to insulate arrayed-electrode; 3 ) Use Inductively Coupled Plas$\mathrm{ma}$ (ICP) to etch silicon in the isolation trenches with the depth of $15 \mu \mathrm{m}$; 4) Do isolation trenches cleaning: a) Use glacial acetic acid and water (1:5) to deal with the isolation trenches; b) Do normal RCA cleaning ( $1 \mathrm{H} \mathrm{NH}_{4} \mathrm{OH}$ : $\mathrm{H}_{2} \mathrm{O}_{2}: \mathrm{H}_{2} \mathrm{O}=1: 2: 7$; 2\# HCI: $\left.\mathrm{H}_{2} \mathrm{O}_{2}: \mathrm{H}_{2} \mathrm{O}=1: 2: 7\right)$; c) Do ultrasonic cleaning by deionized water; 5) Oxidize in the isolation trenches to form a $\mathrm{SiO}_{2}$ insulating layer with the

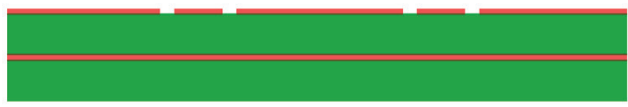

(a) Thermal Oxidating $\mathrm{SiO}_{2}$, etching isolation trenches

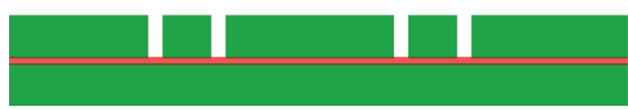

(b) ICP etching isolation trenches

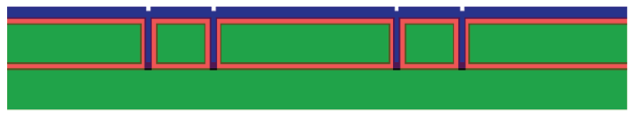

(c) Thermal Oxidating $\mathrm{SiO}_{2}$, depositing polysilicon

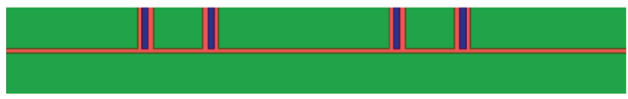

(d) Dry etching, planarization isolation trenches

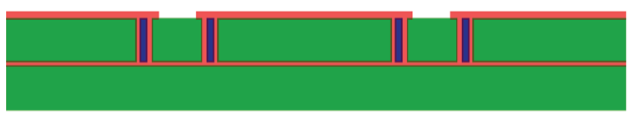

(e) Oxidating $\mathrm{SiO}_{2}$, etching Boron region thickness of $1000 \mathrm{~nm}$; 6) Use polysilicon to refill isolation trenches with the thickness of $2 \mu \mathrm{m}$; The optimum conditions are as follows: polysilicon deposit is controlled at $715-815 \mathrm{~nm} / \mathrm{min}$, and temperature is $590-610$ Degree Centigrade; 7) Oxidize to form a layer of $\mathrm{SiO}_{2} ; 8$ ) Photoetch the region of arrayed-electrode; 9) Boron ions diffuse to form $\mathrm{P}^{+}$arrayed-electrode with the junction depth of $8-12 \mu \mathrm{m} ; 10$ ) Thermal oxidize to form a $\mathrm{SiO}_{2}$ shielding layer with the thickness of $6000 \mathrm{~A}$; 11) Photoetch wire holes and sputter $\mathrm{Al}$ with the thickness of $1.2 \mu \mathrm{m}$ to lead out arrayed-electrode; 12) Alloy to form ohmic contacts; 13) Use Plasma Enhance Chemical Vapor Deposition (PECVD) to deposit a passivation layer with the thickness of $1.2 \mu \mathrm{m}$; 14) Photoetch bonding points and etch $\mathrm{SiO}_{2} ;$ (14) Use Inductivity Coupled Plasma (ICP) to etch silicon with the thickness of $15 \mu \mathrm{m}$ until the layer of $\mathrm{SiO}_{2}$ of the $\mathrm{SOI}$ wafer is exposed.

\subsection{Fabrication PDMS Cover}

PDMS has some advantages, such as low surface free energy $(21.6 \mathrm{dyn} / \mathrm{cm})$, stable chemical properties. There are also some features of flexible, good elasticity and close contact with the silicon substrate. So PDMS is selected as the cover of low voltage CE chip.

During the fabrication, firstly, PDMS monomer and firming agent are mixed by the volume ratio of 10:1. Stir uniformly, and pour it into a flat glass container. Then

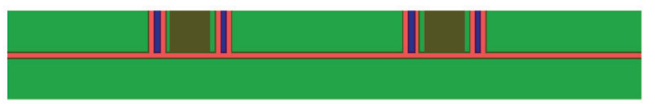

(f) Boron ions implantation

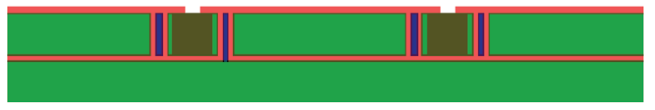

(g) Thermal Oxidating $\mathrm{SiO}_{2}$, etching touch area

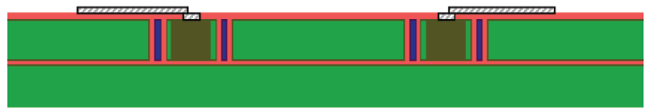

(h) Sputting Al, etching $\mathrm{Al}$ wire

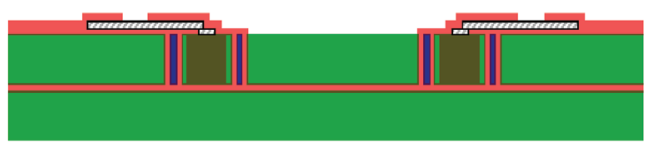

(i) PECVD growning $\mathrm{SiO}_{2}$, etching mental wire

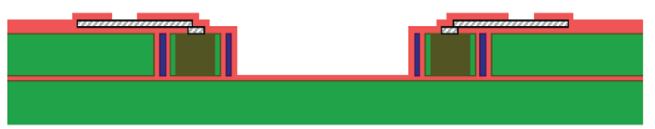

(j) ICP etching micro channel

Figure 3. Processing scheme of low voltage CE chip on SOI. 
pour prepolymer with the thickness of $3-5 \mathrm{~mm}$ on it. Secondly, put the container in the vacuum desiccator to pump for 40 minutes until the air bubbles mixed in it are completely removed. Thirdly, remove the container to a baking oven and then solidify it for 4 hours in the condition of 60 Degree Centigrade. Finally, peel off PDMS lightly from the glass container. Four holes each of which has the diameter of $3 \mathrm{~mm}$ are drilled in the corresponding positions of PDMS as the reservoir. The function for them is to storage sample and waste.

\subsection{Bond and Package}

Because of good adhesiveness of PDMS, it can achieve reversible and irreversible sealing with silicon material. In order to do convenient and completely cleaning, we make reversible sealing with PDMS cover and silicon based bottom part. The specific operation steps are as follows: immerge PDMS cover and silicon based bottom part in the ethanol and distilled water, evaporate to dry ness by nitrogen and then put them together. It is important to align the two parts. The self-fabricated Si-PDMS low voltage $\mathrm{CE}$ chip has good compatibility at the Si-PDMS interface, no leakage, no deformation, good stability and well sealing in the routine operation condition.

Because the number of bond contact in the low voltage $\mathrm{CE}$ chip is far more than that of pins in general cellpack ing, we design PCB to package the self-fabricated low voltage CE chip. The size of the $\mathrm{PCB}$ is $4.43 \mathrm{~cm} \times$ $3.38 \mathrm{~cm}$, and the pads are laid on it to bond with bond contact in low voltage CE chip by Si-Al-wire. Figure 4 shows the photo of Si-PDMS low voltage CE chip with PCB packaging.

\section{Results and Discuss}

\subsection{Inspection of the Fabricated Structure}

The fabricated low voltage CE chip is tested by morphology generation and detection system module of MEMS measurement and micro operation system (Robot Research Institute of Harbin Institute of Technology,

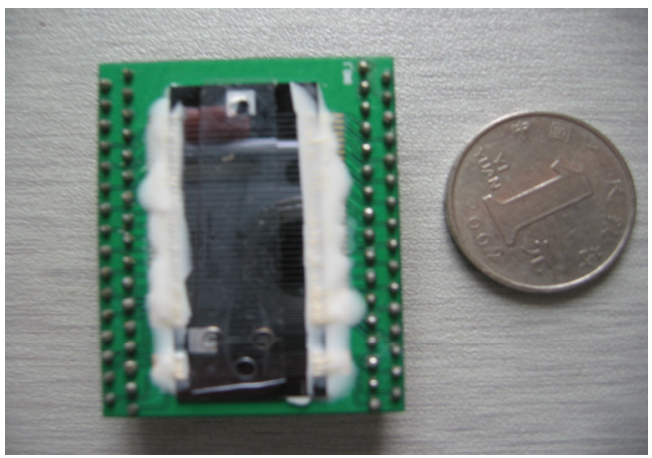

Figure 4. Si-PDMS low voltage CE chip with PCB pacage.

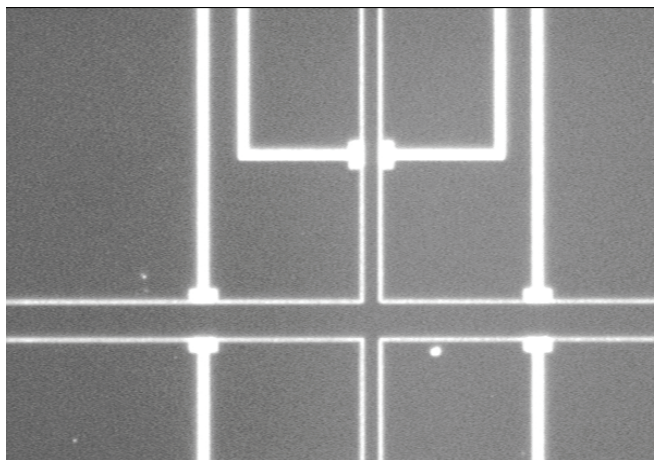

Figure 5. SEM of micro-channel with array-electrodes.

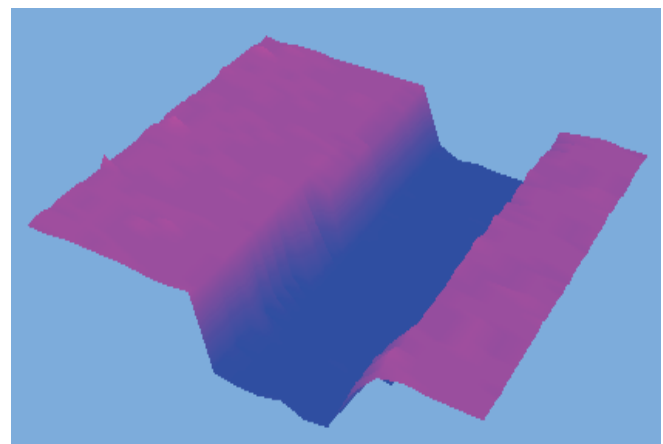

Figure 6. 3-D generation graph of the microchannel.

China). Figure 5 shows the arrayed-electrode at the cross channel. The measured values for width of the separation and injection channel are $83 \mu \mathrm{m}$ and $42 \mu \mathrm{m}$, where the relative error is $3.61 \%$ and $4.76 \%$ respectively. Figure 6 shows the three-dimensional graph of the separation channel. The measured depth value is $15 \mu \mathrm{m}$, which is consistent with our designed value.

\subsection{Electrical Performance Test for Arrayed-Electrode}

In order to test the electrical performance of the fabricated low voltage CE chip, we test and measure the resistance and breakdown voltage of the arrayed-electrode to estimate the insulation and resistance breakdown. We do ergodic experiment on all the arrayed-electrode to measure the resistance of adjacent arrayed-electrode (resistance for arrayed-electrode on 1 st and 2nd, 2nd and 3rd, 3rd and 4th ...until 38th and 39th). The value is infinite for each tested arrayed-electrode pair. It means the isolation trenches are effective, and the insulation between the arrayed-electrode is achieved.

Semiconductor transistor testing instrument is used to test the breakdown voltage of the fabricated low voltage CE chip. We apply testing voltage on the adjacent arrayed-electrode and test all the breakdown voltage on arrayed-electrode (1st and 2nd, 2nd and 3rd, 3rd and 4 th ...until 38th and 39th). The average of the value is 
$306 \mathrm{~V}$, and the standard deviation is $3.41 \mathrm{~V}$. Because the voltage for low voltage electrophoresis separation is only $40-80 \mathrm{~V}$, the breakdown voltage satisfies our needs.

\subsection{Low Voltage Electrophoresis Separation}

\subsubsection{Reagents}

Fluorescein isothiocyanate (FITC) is obtained from Sinopharm Group Chemical Reagent Co. Ltd. (Shanghai, China). Phenylalanine (Phe) and lysine (Lys) are purchased from Shanghai Bio Life Science \& Technology Co. Ltd. (Shanghai, China). 2-(N-Morpholino) ethanesulfonic acid (MES) was obtained from AMRES Co., Hong Kong. All kinds of buffer solutions including MES, His and borate are prepared with redistilled water in $0.1 \mathrm{~mol} / \mathrm{L}$ and diluted in suitable concentration prior to use. All amino acids and stock sample solutions of FITC are prepared respectively in redistilled water to a concentration of $10 \mathrm{mmol} / \mathrm{L}$. Then $10 \mu \mathrm{L}$ FITC solution and $10 \mu \mathrm{L}$ amino acid solutions are mixed and reacted for 12 hours in 25 Degree Centigrade. All the solutions are filtered by $0.2 \mu \mathrm{m}$ filter membrane before being injected into the low voltage CE chip.

\subsubsection{Optimization of Experimental Conditions}

During the electrophoresis separation, separation voltage directly affects the peak time and efficiency of the sample. When the separation voltage is less than $30 \mathrm{~V}$ (that is to say, the field intensity is less than $200 \mathrm{~V} / \mathrm{cm}$ ) the driving force will be too small; while when the separation voltage is greater than $50 \mathrm{~V}$, there is Joule heat effect in the CE chip. Experiment results show that the higher the separation voltage is, the shorter the peak time is. However, if the separation voltage is too high, the peak will come too quickly, and it is disadvantage for sample separation; while the separation voltage is too small, the peak will come too late, and it will lead to long analysis time and worse the analysis efficiency. Finally, we chose $45 \mathrm{~V}$ as the optimization working voltage for low voltage electrophoresis separation.

Switch time is another factor which influences the separation efficiency. If the switch time is too short, movement velocity of sample can not catch up with the velocity of switch time, so the zone of the sample is likely to be cut off into several parts, and cause the baseline turbulence. If switch time is too long, the zone of the sample will accumulate. $2 \mathrm{~s}$ is the optimal switch time for low voltage electrophoresis separation when the separation voltage is $45 \mathrm{~V}$.

\subsubsection{Low Voltage CE Chip Electrophoresis Separation}

Phe and Lys electrophoresis separation experiments are done in the optimal condition: separation voltage: $45 \mathrm{~V}$ $(300 \mathrm{~V} / \mathrm{cm})$; switch time: $2 \mathrm{~s}$. Figure 7 shows electrophoresis spectrum of $10^{-4} \mathrm{~mol} / \mathrm{L}$ Phe and Lys. The peak time

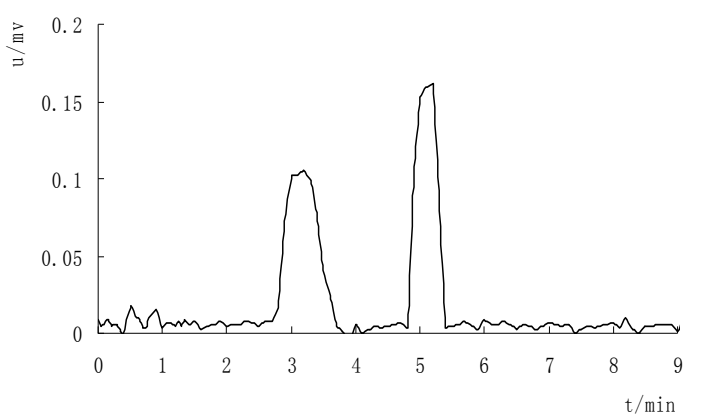

Figure 7. Electrophoresis spectrum of of $10^{-4} \mathrm{~mol} / \mathrm{L}$ Phe and Lys for low voltage CE chip.

for Phe is 6 minuets, while the peak time for Lys is 4 minuets, and the degree of separation is 2.0. From Figure 8 , we got that the self-fabricated CE chip can analysis and test biochemical sample through low voltage electrophoresis separation, It reduces the separation voltage which is thousands of volts to only tens of volts. During the experiments, because the injection volume and time is hard to control, the reproducibility of the testing results is not very good. In future work, we have to improve and enhance the experimental technique.

\section{Conclusions}

We have fabricated a Si-PDMS low voltage CE chip. It contains two parts: one is the silicon based bottom part where there is a cross microchannel and arayed-electrode, and the other is PDMS cover where there are four reservoirs to storage sample and waste. Arrayed-electrode which is used to apply separation voltage is fabricated along the micro channel sidewalls on the SOI wafer. In order to insure the insulation between the arrayed-electrode, isolation trenches are also fabricated. PDMS and silicon based bottom part are reversible sealed to attain Si-PDMS low voltage CE chip. Because arrayed-electrode is the key point of successful electrophoresis of low voltage CE chip, the electrical performance of the arrayed-electrode has been tested, including insulation and resistance breakdown. Results show the resistance between arrayed-electrode is infinite and the average of breakdown voltage is $306 \mathrm{~V}$. Electrophoresis separation experiments have been done to obtain optimum low voltage separation condition: separation voltage is $45 \mathrm{~V}$, the switch time is $2 \mathrm{~s}$ and the Phe and Lys electrophoresis separation is successful.

\section{Acknowledgements}

This research work was supported by Chinese National Science and Technology Committee '863 Plan' (2006AA04Z354) and 'International Cooperation Plan' (2006DFA13510). 


\section{References}

[1] A. Manz, N. Graber, and H. M. Widmer, "Miniaturized total chemical analysis systems: A novel concept for chemical sensing," Sensors and Actuators B, Vol. 7, pp. 244-248, 1990.

[2] N. A. Lacher, K. E. Garrison, and R. S. Martin, "Microchip capillary electrophoresis/electro-chemistry," Electrophoresis, Vol. 22, pp. 2526-2536, 2001.

[3] Y. Jin, G. A. Luo, and R. J. Wang, "Development of integrated capillary electrophoresis chips," Chinese Journal of Chromatography, Vol. 4, pp. 313-317, 2000.

[4] C. Koppmu and A. D. Manz, "Development in technology and application of microsystems," Current Opinion in Chemical Biology, Vol. 1, pp. 410-419, 1997.

[5] J. C. Fister, S. C. Jacobson, and J. M. Ramsey, "Ultrasensitive crosscorrelation electrophoresis on microchip devices, Analytical Chemistry, Vol. 71, pp. 4460-4464, 1999.

[6] J. Qian, Y. Wu, H. Yang, A. C. Michael, "An integrated decoupler for capillary electrophoresis with electrochemical detection: Application to analysis of brain microdialysate," Analytical Chemistry, Vol. 71, pp. 4486-4492, 1999.

[7] Y. Shi, P. C. Simpon, J. R. Scherer, and D. Wexler, "Radial capillary array electrophoresis microplate and scanner for high-performance nucleic acid analysis," Analytical Chemistry, Vol. 71, pp. 5354-5361, 1999.

[8] J. Wang, M. P. Chatrathi, B. Tian, and R. Polsky, "Microfabricated electrophoresis chips for simultaneous bioassays of glucose, uric acid, ascorbic acid, and acetamino-

phen,” Analytical Chemistry, Vol. 72, pp. 2514-2518, 2000.

[9] Y. C. Lin and W. D. Wu, "Arrayed-electrode design for moving electric field driven capillary electrophoresis chips," Sensors and Actuators B, Vol. 73, pp. 54-62, 2001

[10] L. M. Fu and R. J. Yang, "Low-voltage driven control in electrophoresis microchips by traveling electric field," Electrophoresis, Vol. 24, pp. 1253-1260, 2003.

[11] X. Li, Z. Y. Wen, and H. X. Li, "Low voltage separation model and its control system for electrophoresis chip," Micronanoelectronic Technology, Vol. 7/8, pp. 344-346, 2003.

[12] Y. Wu, Z. Y. Wen, and Z. P. Jiang, "Low voltage separation model and discussion for electrophoresis chip," Opto-Electronic Engineering, Vol. 29, pp. 27-33, 2002.

[13] Z. Y. Wen, Y. Xu, and X. Li, "Electric field simulation of separation mode on low voltage electrophoresis chip," Optoelectronic Engineering, Vol. 9, pp. 35-41, 2006.

[14] Y. Xu, Z. Y. Wen, and X. Li, "Fluidic field simulation of separation mode on low voltage electrophoresis chip by ConventerWare," Chinese Journal of Sensors and Actuators, Vol. 8, pp. 1070-1075, 2007.

[15] Z. Y. Zhang, S. L. Xu, and Y. K. Liu, "A Study on silicon deep etching technology," Microelectronics, Vol. 2, pp. 519-521, 2004.

[16] Y. Wu, Y. Q. Jiang, and Z. Y. Wen, "Development of polycrystalline silicon micro-electrode array on silicon substrate," Semiconductor Optoelectronics, Vol. 4, pp. 317-319, 2005. 